\title{
A MODERN APPROACH to KNEE INJURIES
}

\author{
By J. A. C. PRINSLOO, B.Sc. (Physio.)(Rand)
}

The treatment of knee injuries has always been mainly the responsibility of the physiotherapist, especially in orthopaedic and rehabilitation units, once corrective surgery has been done. Although most of the old principles still hold, recent advances in the actual surgery have been so great, that the consequent and concomitant physiotherapy treatment has to be modified to meet demands and ensure lasting results. The treatment should be approached intelligently and discretion used in each individual case in order to obtain maximum results. Wrong or bad treatment can often do more harm than no treatment at all and even reverse the corrective and curative processes.

Movements of the Knee Joint

Flexion is produced by the hamstrings, i.e. biceps, semitendinosus and semimembranosus, as well as gastrocnemius, popliteus, plantaris, sartorius and gracilis. Popliteus initiates flexion by laterally rotating the femur on the tibia, which can then also be assisted by semitendinosus, semimembranosus, sartorius and gracilis.

Excessive flexion is checked by the contact of the leg and thigh, as well as the structure of the tibial and femoral condyles; also partly by tension in the ligamentum patellae and the posterior cruciate ligament.

Extension is produced by quadriceps and terminated by medial rotation of the femur on the tibia, which is the very important function of vastus medialis.

Excessive extension is prevented by the tension of the posterior part of the capsular ligament, the medial and the lateral collateral ligaments and the posterior cruciate ligament, as well as the hamstrings and gastrocnemius.

Rotation, unassociated with flexion or extension, is most pronounced with the knee flexed to a right angle.

Medial rotation is produced by popliteus, semitendinosus and possibly semimembranosus, gracilis and sartorius. It is checked by the anterior cruciate ligament.

Lateral rotation is produced by biceps and controlled by the medial collateral ligament.

The extended position of the knee in the erect posture is maintained mainly by gravity and as long as the iliofemoral ligament is tense, as this prevents lateral rotation of the femur. Thus flexion of the hip and the knee have to occur concomitantly. Yet, vastus medialis is the most important single and active muscular factor concerned in obtaining "locking" of the knee.

\section{Routine Examination of the Knee}

The following points of examination, if followed consistently, would show up most facets of internal derangement of the knee, with associated and concomitant injuries and is supplemented by stress radiography where any doubt exists or to confirm a diagnosis.

1. Movement. (Active and passive).

(a) Extension to the full. Loss of extension is usually painful and the site of pain is important.

(b) Flexion. Loss of flexion is not of too much importance as this is the first reaction to even minor trauma.

2. Crepitation.

(a) Patello-femoral, found by moving the patella in all the directions.

(b) Joint, found by normal passive movement whilst palpating the joint at the same time.

3. Collateral Ligaments.

Laxity of both the medial and the lateral ligaments can be demonstrated by passive medio-lateral stress of the fully extended knee.

4. Cruciate Ligaments.

The patient is supine with the knee flexed to a right angle and fully relaxed. The tibia is moved passively on the femur. Undue laxity of the anterior cruciate ligament is demonstrated by "drawer forwards" and trauma to the posterior cruciate ligament is shown up by "drawer backwards"

5. Tibial Rotation.

(a) Medial rotation. The patient is in supine with the hip and knee fully flexed. Using the foot as a lever, the tibia is medially rotated by a circumduction movement which ends in nearly full extension, while the joint is palpated. This is used specifically to investigate the condition of the posterior portion of the medial meniscus.

(b) Lateral rotation. This is the reverse of the above procedure and is used to examine the posterior portion of the lateral meniscus.

(c) Circumduction. This is done with the knee in 20 degrees flexion throughout and both hands palpate the joint while a small circle is described. This technique is used to detect lesions in the anterior portions of the menisci.

6. Manipulation Under Anaesthesia.

This is seldom used as pain is of great diagnostic value and thus it would only be a last resort.

7. Pain.

This is tested by patient's symptoms as well as by pressure palpation for tenderness of the collateral ligaments and the jointline. Exact localization of the pain and tenderness can be of diagnostic value. A bduction and adduction of the tibia will pinch the respective menisci (if ruptured) and cause pain.

8. Reflexes.

Knee jerk, ankle jerk, plantar response etc. are routinely tested.

9. Effusion.

This is established by the "patella tap" and joint fluctuation.

10. Gait, general appearance and systematic reactions (if any, to determine septic focus).

\section{TRAUMATIC CONDITIONS OF THE KNEE 1. SOFT TISSUE INJURIES.}

\section{A. Traumatic Synovitis.}

Normally synovial fluid, $\frac{1}{2} \mathrm{cc}$, is produced by special cells in the synovial membrane. It consists mainly of mucin, which is alkaline, and fulfils the function of protection against excessive acidity. Further it lubricates the joints, is concerned with the nutrition of the joint structures and the rich blood supply of the synovial membrane dissipates excessive heat and metabolytes. The production of synovial fluid is stimulated by the normal movement of the joint.

On trauma, the synovial membrane reacts twofold:

1. There is increased activity of the synovial cells with the resultant increase in mucin secretion and eventual hypertrophy of the cells.

2. A general reaction also takes place, which consists of vasodilatation with extravasation of plasma, obliteration of lymphatics, increased acidity and even precipitation of fibrin with resultant granulation.

Traumatic synovitis of the knee is in actual fact a symptom accompanying all acute knee injuries and cannot be diagnosed as a condition as such until all other possibilities have been eliminated.

It consists of swelling of the knee joint with "floating" of the patella which can be demonstrated by the "patella tap". Usually it appears a few hours after injury, offers little resistance to pressure and is not accompanied by a general reaction.

\section{Treatment:}

Once all other possibilities have been eliminated as a result of a complete investigation, a simple traumatic 
synovitis is treated with a compression bandage and nonweight bearing exercises to the quadriceps. Ice applications can be used to aid circulation, but conscientious exercise is of prime importance. If the effusion is particularly gross, it is aspirated before applying the bandage.

Chronic synovitis is difficult to treat as it is usually as a result of neglecting exercise in the initial stage and this can never be compensated for. At its best it takes several weeks and even months of concentrated exercise to clear up the condition and restore the muscular balance in a weak and unstable knee and at its worst it can lead to post-traumatic osteoarthritis.

\section{B. Traumatic Haemarthrosis.}

It is very seldom that a simple haemarthrosis without accompanying trauma to bone, capsule, ligaments or other soft tissues, occurs. If all other possibilities have been eliminated, it is treated by immediate aspiration, application of a compression bandage and immobilization for ten to fourteen days to prevent further haemorrhage. After that active knee exercises are started.

Usually a haemarthrosis is the result of trauma to the synovial membrane, ligaments or a fracture of the tibial spine. In this case there is little damage and haemorrhage and virtually no clotting of blood. Usually this type has a good prognosis and is treated by aspiration and rectification of causative trauma. Subchondral fractures may not be visible on radiography, but can be diagnosed by virtue of the excessive fat globules floating on the surface of the aspirated fluid or by means of repeat radiography after ten to fourteen days. If it is suspected, weightbearing would be deferred until confirmation or otherwise.

If the soft tissue damage is much more extensive, there will be excessive haemorrhage with clotting. This stimulates mucin production with a resultant effusion and obliteration of the lymphatics. Organization and eventual adhesion formation may take place, greatly impairing function and the prognosis is not rosy.

Clinically a haemarthrosis can be distinguished from an effusion in that it occurs virtually immediately after injury (of course there are varying degrees and combinations of both), there is usually acute pain and even systemic reactions like a raised temperature and the swelling has a higher resistance to pressure than a simple effusion.

It may be of use here to mention what the ideal compression bandage should be like to fulfil its main function, namely to control and prevent effusion. It is usually applied by the surgeon, immediately after completion of the operation and before the torniquet is removed or after aspiration of the joint. At least a pound of cotton wool is used and it is applied in three layers, each secured by a tight domette bandage and stretching from the ankle to the groin. This ensures full control of the soft tissues and no knee movement will be possible. Thus static quadriceps exercises can be undertaken safely without fear of knee-movement.

\section{LIGAMENTOUS INJURIES.}

The ligaments constitute the passive control against injury by excessive movement in the joint and they also determine the direction in which muscle action will be transmitted to the moving surfaces. It is a well-known fact that injury to any one isolated ligament will cause very small instability in a joint surrounded by normal musculature and not subjected to excessive muscular work. Thus laxity and instability result from trauma to more than one ligament with or without concomitant meniscal injury. The only exception possibly is the medial collateral ligament.

\section{A. Medial Ligament.}

\section{Partial Rupture or Sprain.}

This usually occurs as the result of a relatively minor external rotary strain whilst the knee is in slight flexion.

It can be recognized by swelling over the medial aspect of the knee with vague tenderness. This tenderness can be localized by palpation, usually near the femoral attachment and can be reproduced by lateral rotation in slight flexion. The joint is stable on passive abduction and a synovitis or other complications absent in a simple tear. Where complications are present, a full diagnosis is impossible and the lesion is treated primarily with the possibility of a later investigation.

\section{Treatment}

A local injection of anaesthetic into the area of most tenderness, followed by an elastic adhesive bandage often affords dramatic relief and quadriceps drill and even weightbearing can be instituted immediately in these simple cases.

In the case of e.g. effusion as a complication, the above treatment is given, plus a compression bandage and immediate non-weightbearing quadriceps drill to control and decrease the synovitis. Once the swelling is down and the muscles can cope, weightbearing is started. A full diagnosis, e.g. meniscal tear, can only be made should the joint not stand up to the strain of sport etc. again.

Pelligrini Steida's Disease.

In some of these cases of partial rupture of the medial ligament the symptoms (pain and decreased ROM) seem to increase, even in the presence of diligent physiotherapy treatment. This is due to post-traumatic para-articular ossification or so-called Pelligrini Steida's disease. This condition is said to result from calcification and resultant ossification at a fertile site, usually an area of oedema or haematoma and is thus more frequently found in cases of direct trauma to the medial ligament, like a blow, rather than indirect trauma as described above.

On examination in the early stages there is gross weakness and loss of flexion (even passive), severe pain, localized tenderness and a vague thickening of the deep tissues. At a later stage the symptoms are less acute, but a bony prominence can be felt medially. Ultimate diagnosis can be made by means of radiography, usually visible only after six weeks, but the above can be clear cut enough.

\section{Treatment.}

1. Mainly by preventing it by accurate diagnosis and correct treatment or by recognizing it in an early stage and avoiding passive movement, massage or manipulation, all of which tend to encourage the formation of heterotopic bone.

2. Once a diagnosis has been made, all vigorous exercises are avoided, but gentle quadriceps drill and flexion exercises up to the limit of pain are done during the active phase, which may last from six to eighteen months. Surgical intervention is diliterious in the early stage. In some cases the disease is selflimiting and resorption may even take place.

3. In most cases surgical excision of the heterotopic bone is necessary, as well as severing of all adhesions, once the disease is no longer active. A compression bandage is applied and active knee movements started within 3-4 days.

\section{Complete Rupture.}

This is a very disabling injury as, apart from the instability caused in the knee joint, it is usually accompanied by trauma to the cruciate ligaments, rupture of the capsule, meniscal injury and fracture of the lateral condyle of the tibia. It is usually caused by direct trauma and followed by severe pain and disability and a sensation of "tearing" in the joint. It may also be misleading as to seriousness, as a haemarthrosis may be absent in the case of a torn or ruptured capsule, which allows the blood to escape from the joint. A complete rupture can only be finally diagnosed after abduction "stress" radiographs.

Treatment.

(a) Conservative.

This is followed in cases where the lesion is not quite complete or operative measures are contra-indicated, e.g. in the presence of skin injury which will delay operation for longer than ten days.

The treatment consists of aspiration of the joint in the case of gross synovitis or haemarthrosis and then the application of a compression bandage, sometimes reinforced e.g. by Kramer wire or a posterior splint. Quadriceps drill is given 
in these cases with extreme care and always within the limit of pain, avoiding any movement whatever and stopping if there is the slightest increase in symptoms. Once the swelling has subsided, a skintight walking plaster is applied and extensive quadriceps drill can be commenced. Weightbearing is allowed a few days later, always with the understanding that the symptoms have subsided sufficiently and the musculature can cope adequately. Plaster immobilization is maintained from six to twelve weeks and maintenance and increase of strength of all muscle groups around the knee during this time is the one single factor which can determine complete recovery or otherwise. Once the plaster is removed, mobilization of the knee is commenced and correct and even protective gait must be taught. Quadriceps exercises should be kept up for a few months.

\section{(b) Operative.}

This is usually resorted to in the presence of other disabling injury, e.g. cruciate or meniscal damage, or where the single injury to the medial ligament is so extensive and causes such instability, that it may lead to more damage of other structures controlling the stability of the knee joint. Thus in most cases it will be part of a general reconstructive operation, which is discussed later. Here the knee will be immobilized for four to six weeks, during which time quadriceps drill and general maintenance exercises are done followed by mobilization and weightbearing.

\section{B. Lateral Ligament.}

This ligament is of relative unimportance in stabilizing the knee joint. It is reinforced by the iliotibial band and biceps and lax in flexion, and thus not susceptible to rotationin-flexion strains. Consequently an isolated complete rupture of the ligament rarely occurs and it is usually part of gross trauma due to great violence, e.g. powerful adduction of the leg on the thigh. In most cases it is accompanied by a traction injury and subsequent gross damage to the lateral popliteal nerve or avulsion of the head of the fibula, although both can escape.

\section{Treatment.}

As regards the ligament, treatment can be conservative or operative, depending on the treatment of the concomitant trauma. As regards the nerve lesion, the prognosis is much worse. Traction lesions especially show extensive damage and very little recovery. The best approach seems to be exploration and suture where and as early as possible as this affords the best recovery possibilities.

\section{Cruciate Ligaments.}

These constitute the factors of internal stability of the knee. The anterior cruciate is of more importance clinically as it is usually involved in total disruption of the medial ligament and can be the only ligamentous injury associated with tears of the medial meniscus.

\section{Anterior Cruciate.}

In a careful analysis of the anterior cruciate, it has been found to be tense through the whole range of flexionextension. It is true that the greatest tension is in extension and affects mainly the anterior fibres; in semiflexion the ligament is most lax, although the middle fibres are still more tense and in full flexion the postero-lateral fibres are tense.

The mechanism of injury is usually rotary and associated with medial meniscal injury, e.g. forced flexion or extension with the tibia fixed or the above in the presence of previous internal derangement (usually menicsal tear) which prevents tibial rotation in the last bit of the ROM. The latter can be a sudden rupture or slow stretching and attenuation in the more chronic cases. Abduction injuries are less common, but more important, as it occurs only after rupture of the medial ligament has taken place. The injury can be complete, with the ligament intact, but bone avulsed, (usually from the inferior attachment to the tibial spine area) or complete with rupture of the ligament itself (usually near the superior attachment to the femoral condyle) or partial rupture and attenuation due to continuous minor traumata.

Acute rupture of the anterior cruciate is usually associated with a medial meniscal tear (with or without recurrent locking) or with medial ligament rupture with or without fracture of lateral tibial condyle.

Often the diagnosis of a ruptured anterior cruciate ligament is difficult as it may be masked by concomitant trauma or complications, e.g. ruptured medial ligament, haemarthrosis, synovitis, acute pain, tenderness and muscle spasm. Unless the patient is seen very soon after the injury, it is best to examine the knee after aspiration and even anaesthesia. Laxity can be demonstrated by the "drawer forwards" sign. It has to be compared with the sound side. The "drawer forwards" sign is usually "minimal" on isolated rupture of the anterior cruciate and "maximal" when associated with rupture of the medial ligament. Usually radiography is negative, except where an avulsion fracture of the tibial spine is present.

\section{Posterior Cruciate.}

As for the anterior cruciate, the posterior is said to be taut throughout flexion-extension, the tension changing from the anterior to the posterior fibres as the joint moves from flexion to extension. Thus it will prevent hyperextension and also control medial rotation of the tibia in flexion.

Usually rupture of the posterior cruciate is found as part of extensive trauma to the knee and rarely as an isolated injury. The mechanism of injury is usually the tibia being driven posteriorly on the femur in the flexed knee. The type of rupture follows the pattern already described for the anterior cruciate ligament. It can be diagnosed by the "drawer backwards" sign.

\section{Treatment.}

As has been stated previously, rupture of any one ligament only, causes hardly any disability, especially in the presence of good musculature around the knee and where no strenuous demands are made on it, e.g. competitive sport, mining, etc. In cases where there was great laxity and disability, several types of substitutions were used, e.g. Hey Grove's fascialor Smillie's meniscal substitution, but these seemed to have no lasting effect. In cases of severe laxity and derangement even arthrodesis was used.

Then in the last decade Lindemann's Heidelberg operation was developed and has been used increasingly with success. Briefly it is as follows:

\section{For Anterior Cruciate.}

The knee is opened by a fairly extensive antero-medial incision and the joint examined. If necessary, a medial menisectomy is performed. The stumps of the ruptured anterior cruciate is excised. Gracilis is divided near its insertion and mobilized by blunt dissection to more or less a third way up so that the line of pull will be from the posterior midline. It is then passed through the lateral part of the posterior capsule, along the intercondylar notch to appear in the anterior part of the joint. A hole is tunneled from medial to the attachment of ligamentum patellae on the tibia, to emerge at or anterior to the inferior attachment of the anterior cruciate, gracilis is passed through the tunnel and one and a half inches of tendon is secured to the tibial periosteum and ligamentum patellae insertion with moderate tension. Bone chips are punched into the tunnel to ensure adequate fixation. The wound is closed in layers. A well padded long leg plaster is applied and suspended from a Balkan frame for a few days by means of wire loops.

Isometric contractions of all the leg muscles can be started immediately and should be done conscientiously, but with adequate rest, during the waking hours. Non-weightbearing ambulation is allowed within 5-7 days. The plaster is removed after 5-6 weeks and full strengthening and mobilization can start. Here it is of prime importance to strengthen all the supporting musculature of the knee joint. Teach correct and protective ambulation, i.e. "bracing" the knee on weightbearing and avoiding sudden twists.

Medial Ligament.

Usually this is found to be lax and repaired at the same time. If there is only slight laxity, it is sutured, or if warranted an O'Donoghue's distal shift operation can be performed. 


\section{For Posterior Cruciate.}

The approach is similar as for the anterior cruciate. Gracilis is severed and loosened and then passed through a tunnel drilled through the medial femoral condyle from just distal to the adductor tubercle to emerge in the anterior compartment of the knee joint at the point where the posterior cruciate attaches to the medial femoral condyle. The tendon is passed medial to the anterior cruciate to enter the posterior compartment of the joint. It is held there with a Kocher's forceps, the incision is closed and the patient turned onto his face. A second incision is made in the midline of the popliteal fossa and the Kocher's forceps with the tendon, located. The posterior capsule and periosteum is cleared on the back of the tibia for one inch and the gracilis tendon securely sutured to the periosteum and the capsule, with the knee in thirty degrees flexion. The wound is closed and the after treatment is the same as for the anterior cruciate.

Combined Repair.

Should both cruciates be ruptured, gracilis is used to substitute the anterior and semitendinosis the posterior. Repair of the medial ligament, if necessary, is done before the first incision is closed.

Although there is still appreciable laxity in the joint after this operation, it is extlemely stable during strenuous activity and more awareness of tension exists than in fascial transplants.

\section{MENISCAL INJURIES}

The menisci or semilunar cartilages fill in the incongruity between the femoral and tibial condyles to provide more acceptable and functional joint surfaces. It is said that the movements of rotation take place below the menisci and flexion and extension take place above the menisci. Removal of the menisci does not seem to impair the function of the knee joint, again provided that the surrounding musculature is in good condition.

\section{(a) Medial Meniscus.}

The horns of the medial meniscus are wide apart but firmly attached to the tibia in the non-articular intercondylar area. The periphery or arch is loosely attached to the tibia by means of the coronary ligament (part of the capsule) and firmly attached to the femur by means of the deep fibres of the medial collateral ligament. Thus the medial meniscus is especially mobile on the tibia in rotary movements and suffers rupture during rotational stress because of this mobility of its arch which lacks muscular control. During rotation the horns of the meniscus move with the tibia and the arch with the femur, leading to distortion and rendering it liable to injury.

\section{(b) Lateral Meniscus.}

It is more circular in shape with the anterior and posterior horns much closer together and also attached to the intercondylar area of the tibia. The arch is loosely attached to the tibia by means of the coronary ligament, but the ligaments of Humphrey and Wrisberg, and especially the potterior superficial fibres of popliteus, actively control the arch of the lateral meniscus during rotation. Popliteus acts as an external rotator of the femur from extension i.e. it initiates flexion, but its main function is believed to be control of the lateral meniscus by preventing it from moving medially to a position where it can be crushed, as the knee unlocks for fiexion.

The mechanism of injury is similar for both menisci, namely forced rotation, but the lateral meniscus is less liable to this and is injured more often as a result of direct violence. Meniscal injuries occur mainly in young healthy males who play active sports like football and rugby or work in mines which involves strenuous work in confined spaces on fully flexed knees. Usually the tearing is as a result of forced rotation of the femur on a fixed tibia with the knee in flexion. By taking a detailed history of the mechanism of injury, resultant incapacity, course and complications or resultant disability from the patient, will usually give a fairly clear indication as to the trauma. By a careful clinical investigation along the lines already described, a diagnosis can usually be made.

One should never lose sight of the natural processes of degeneration that set in in the knee joint after the second decade and always judge what is the relative "normality" of the joint before reaching a conclusion and deciding on treatment.

\section{Treatment}

Minor peripheral tears are sometimes treated conservatively along the lines of a medial collateral ligament rupture, but these usually give rise to symptoms later, so that operative procedures are followed in nearly all cases. This is decided on after persistant locking and persistance of symptoms, notwithstanding effective conservative treatment.

After menisectomy, a compression bandage is applied and the patient is nursed with the feet elevated. Static quadriceps exercises are started immediately and partial weightbearing allowed after 5-7 days. The stitches are removed after ten days and gentle mobilisation of the knee (always using pain as a criterion and avoiding causation of any symptoms that are prolonged after treatment) is started. After three weeks, strong weight bearing exercises can be started and ideally the patient should be able to return to work after 6-8 weeks and sport after three months.

\section{DISLOCATION.}

Complete dislocation of the knee occurs very rarely and then as a result of extreme violence, directly, to the lower end of the femur with the tibia firmly fixed, or to the upper end of the tibia with the femur fixed. The type of dislocation will depend on the direction of the violence. Dislocation as a result of rotary violence is even more rare.

Dislocations of the knee are classified according to the relative position or movement of the tibia to or on the femur. Further they can vary in degree from incomplete to complete (the latter is of purely academical interest) and be of the closed or open variety. The latter will be complete with extensive soft tissue damage.

(Please see next page)

\begin{tabular}{l}
\hline ORTHOPAEDIC MECHANICIANS \\
Manufacturers and Suppliers of: \\
ORTHOPAEDIC APPLIANCES, ARTIFICIAL \\
LIMBS, TRUSSES, SURGICAL CORSETS, \\
URINALS, ARCH SUPPORTS, COLOSTOMY \\
BELTS, ELASTIC STOCKINGS, ANKLE \\
GUARDS, WRIST GUARDS, ELBOW GUARDS, \\
KNEE GUARDS, LIGHT DURAL CRUTCHES \\
FOR CHILDREN, WOODEN CRUTCHES, AND \\
METAL ELBOW CRUTCHES. \\
Phone 23-2496 \\
312 Bree Street, Johannesburg \\
\hline
\end{tabular}


All types will involve the collateral ligaments, cruciates, capsule, popliteus, gastrocnemius and vastus medialis to a greater or lesser extent. The latero-medial type can involve the lateral popliteal nerve and then especially the medial dislocations; often rupture of the skin and underlying soft tissues occur and then a closed reduction is incomplete due to the obstrusion of soft tissues into the joint. Anteroposterior dislocations usually damage the posterior capsule and cruciates whilst the collateral ligaments may escape with relatively little damage and more often than not the popliteal artery is in grave danger.

Diagnosis is easy due to the gross trauma and deformity and from the history. Radiography is essential to eliminate or establish concomitant fractures.

\section{Treatment.}

Reduction under anaesthesia should be undertaken as soon as possible, once the state of the circulation has been found satisfactory. Arterial insufficiency due to rupture or thrombosis or spasm of the popliteal artery requires immediate exploration and this then has priority in treatment with the result that reduction may be deferred until the circulation has been restored to satisfaction. Failure of this would necessitate midthigh amputation. Primary suture of a torn popliteal nerve may be undertaken if an open reduction is necessary or in the case of an open dislocation or it may be left till a later date. Once more, traction lesions have the worst prognosis.

A compression bandage with backsplint or Kramer wire reinforcement is applied (in slight flexion where the posterior capsule is involved). During the first 48 hours, especially, the circulation has to be checked frequently. Once this is satisfactory, the joint is aspirated and another compression bandage applied.

Provided there are no complications, quadriceps exercises can be commenced after a week once a plaster spica has been applied. Weightbearing can be gradually started after 6-8 weeks on application of a skintight plaster. Immobilization is usually maintained for three months. During this time conscientious exercise of all the leg musculature is of paramount importance and may actually determine the end result. At the end of three months most soft tissues will have healed and mobilization may begin. Often there will be excessive fibrous tissue and even hererotopic bone formation, which must be taken into consideration during mobilization. In cases of severe internal derangement this may actually constitute additional support. In most cases functional recovery (with or without aiding apparatus, e.g. for dropped foot) can be achieved with adequate strengthening of the musculature, but flexion will invariably be limited.

\section{OSTEOCHONDRITIS DISSECANS}

This will be mentioned very briefly as some authorities hold that the condition does occur as the result of trauma. This is a condition where a fragment of articular cartilage becomes loosened, sclerosed and eventually separated off to form a loose body in the joint. The main symptoms are vague pain, locking and wasting of the quadriceps. Diagnosis in the early stages is difficult due to the vagueness.

\section{Treatment.}

In young adults conservative treatment has been used, more on an experimental basis. The knee is immobilised and no weightbearing permitted for three months. If healing has occurred, the joint is immobilized for a further three months and then gradual weightbearing and mobilization started.

Most cases are treated operatively by means of arthrotomy, whether before or after separation of the fragment, depends on the severity of the symptoms as well as the views of the surgeon. After treatment is like that for a menisectomy.

The results will depend greatly on the size of the fragment(s) and the area affected. If a large portion of the weightbearing area is affected at an early age, early and gross arthritic changes are to be expected.

\section{FRACTURES.}

In general, those fractures that involve the knee joint directly, are fractures of the tibial spine, of the tibial plateau (involving the lateral only or both condyles) or subchondral fractures of the patella and condyles. As there is such a large variant in the severity of these and the concomitant and complicating soft tissue injuries associated with them, which in turn gives rise to very varied scope of treatment that could fill another paper, they will also be mentioned very briefly.

The tendency in treatment where fixation and nonweightbearing are necessary, today is for skeletal traction by means of pins and the use of the so-called Perkins bed which still allows movement of the knee. In older people especially, the tendency is to stress mobility even if weightbearing is not allowed.

\section{PHYSIOTHERAPY IN KNEE INJURIES.}

The quadriceps is by far the most important mechanism that is involved in knee injuries and the subsequent return to function, but one should always aim for a balanced action of the supporting muscles and not lose sight of the importance e.g. of popliteus in initiating flexion, or that of the hamstrings in preventing hyperextension.

The orthograde posture is unique to man and a relatively recent biological acquisition. It is a delicate mechanism that is easily deranged by injury. Loss of volume, tone and control in itself constitutes a disability. Quadriceps insufficiency leads to intra-articular trauma as the joint cannot cope with the normal stresses and strains and this in turn leads to further weakening of quadriceps. The only way to cope with this vicious cycle is to start non-weightbearing exercises that will build up strength and tone sufficient to cope with weightbearing. The reflex inhibition of quadriceps after trauma can resemble a flaccid paralysis. Thus after trauma and operation it is of prime importance to institute active quadriceps contractions (isometric or isotonic as the case may be) immediately, not only to preserve strength, but also to maintain the kinaesthetic sense. Heat, ice and massage are only complementary measures to increase the circulation.

Quadriceps exercises contra-indicated in:

1. Open wound treated by primary suture.

2. Haemarthrosis.

3. Acute sepsis.

These all require an initial period of rest.

Functionally, quadriceps can be divided into two components, namely:

1. Vastus lateralis, vastus intermedius and rectus femoris which cause extension of the knee.

2. Vastus medialis, responsible for the last 10-15 degrees of extension and thus the all important "locking" of the knee. Even if there is no internal derangement, mal-function of this will cause an unstable knee. It is also the site of first and most marked wasting.

The following are some points of application to remember when working for the optimum in recovery of the musculature surrounding the knee joint:

1. The all or none law that governs contractile units.

2. One has maximal mechancial efficiency when the muscle fibre starts to contract from the fully stretched position.

3. To increase power (of first importance here) exercises of a high resistance, low repitition rate are best.

To increase endurance, exercises of low resistance, high repetition rate give the best results.

4. Co-ordination.

(a) Speed of contraction to prevent injury.

(b) "Timing", i.e. the importance of actions other than the prime mover, thus proprioception.

5. Dosage.

(a) Weightbearing too early gives rise to increased symptoms and may even be detrimental.

(b) Develop power and then endurance.

6. Relaxation between individual exercises and sessions is important especially in prolonged atrophy.

7. Progression can be in time and work done. If necessary, one can start with Faradism to inttiate contraction where the kinaesthetic sense is lost, but voluntary contraction is the most important. 
8. Variety. Exercises should be specific for the knee, but also general, especially when the patient is still confined to bed, and varied enough to hold the interest.

From the above it will emerge that one has to have at least one session per day (and more short sessions in the early stages) to concentrate on individual exercises for the specific condition. Here one has to stress the importance of regular exercise throughout the day of the principal muscle groups, namely quadriceps, hamstrings, foot pumping and gluteal contractions as well as isolation of vastus medialis and popliteus, which is the patient's responsibility. Fiom the third day on most patients can participate in class work, mainly aimed at general maintenance, which is extremely valuable from the point of view that it stimulates interest, encourages patients to work on their own, allows for therapeutic games in the later stages and of course economises the use of the physiotherapist's time.

Physiotherapy can play a very important part in the rehabilitation of knee injuries, especially by means of conscientious exercise, provided it is applied accurately and with intelligence. Ideally one would want to start training the patient pre-operatively where possible. Firstly this acquaints the patient with the exercises expected post-operatively and their relative importance in the rehabilitation programme. Secondly it provides the physiotherapist with some norm whereby to measure progress. Thirdly it can prevent the extremely rapid loss of kinaesthetic sense, loss of tone and atrophy which is peculiar to quadriceps. In more chronic cases it has become practice to treat patients on an out-patient basis for a week prior to operation to teach exercises and expected ambulation necessary post-operatively.

Individual exercises will be left to the choice of each physiotherapist, as well as a rehabilitation programme, as this depends largely on the set-up of each department, personal ability and surgeons concerned in the case. In conclusion it can be mentioned that P.N.F. techniques have been most effective for individual treatment. There is a good variety of strengthening and mobilizing techniques which can be used in conjunction with ice to ensure speedy and optimum rehabilitation.

Note.

The author wishes to thank Prof. G. T. du Toit and Dr. I. S. de Wet of the Pretoria Orthopaedic Hospital for their help and encouragement in the preparation of this article.

\section{A PRAYER}

The following prayer should be of great help to people as they grow older; and it contains much concentrated wisdom!

Lord, Thou knowest better than I know myself that I am growing older, and will someday be old.

Keep me from the fatal habit of thinking I must say something on every subject and on every occasion.

Release me from craving to straighten out everybody's affairs.

Make me thoughtful but not moody; helpful but not bossy.

With my vast store of wisdom it seems a pity not to use it all, but Thou knowest, Lord, that I want a few friends at the end.

Keep my mind from recital of endless details; give me wings to get to the point.

Seal my lips on my aches and pains; they are increasing and love of rehearsing them is becoming sweeter as the years go by. I dare not ask for grace enough to enjoy the tales of others pains, but help me to endure them with patience.

I dare not ask either for improved memory; but for a growing humility, and the lessening cock-sureness when my memory seems to clash with the memories of others.

Teach me the glorious lesson that occasionally I may be mistaken.

Keep me reasonably sweet; I do not want to be a saintsome of them are so hard to live with.

But a sour old person is one of the crowning works of the devil.
Give me the ability to see good things in unexpected places and talents in unexpected people.

And give me $O$ Lord, the grace to tell them so.

AMEN

\section{A STUDENT OUT-PATIENT CLINIC}

The out-patient department at the Johannesburg Hospital comprises womens' and mens' treatment rooms, staff gymnasium, students gymnasium and students out-patient clinic.

At the clinic the 3rd and final year students treat a crosssection of out-patients under the supervision of a lecturer in physiotherapy. They get experience in individual treatments by massage, electrotherapy, passive movements, traction, the application of heat and cold and individual exercises. Group exercises and heavy rehabilitation is done in the gynmasium.

It was suggested that it might be of interest to physiotherapists to see what types of patients are treated here.

An analysis of patients treated from February, 1965 to February, 1967 revealed the following:

$\begin{array}{llllll}\text { Surgical out-patients } & \ldots & \ldots & \ldots & \ldots & 138\end{array}$

$\begin{array}{llllll}\text { Medical out-patients } & \ldots & \ldots & \ldots & \ldots & 67\end{array}$

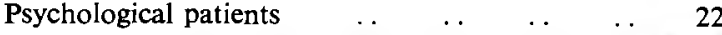

$\begin{array}{llllllll}\text { TOTAL } & \ldots & \ldots & \ldots & \ldots & \ldots & \ldots & 227\end{array}$

It is of interest to note the high incidence of psychogenic cases sent for physiotherapy. Of these seven were receiving psychiatric treatment. Further analysis of this group shows the site of pain or paralysis to be distributed as follows:

The neck-7, Lumbar region-1, arms-3, leg or knee-3, feet-3, Hands- 4 .

One had "general pains".

The "treatment" requested was usually some form of heat (wax, S.W.D.), massage and exercises.

The surgical group was the largest as it included trauma and orthopaedics. Further analysis of this group included:

General surgical (amputations, post-mastectomy oedema, stress incontinence etc.)-13, Fractures - 25, hands-19, soft tissue lesions - 19 , feet-25, spinal lesions- 37 .

The fracture patients were usually referred for treatment of complications such as stiff joints or nerve lesions or residual pain and swelling. Very few recent injuries are seen.

The "hands" were the usual cut tendons, septic fingers, post-traumatic stiffness.

Soft tissue lesions were mainly tendonitis. The foot patients were all women suffering from pain and weakness of the intrinsic muscles.

Of the spinal lesions 8 were cervical, one thoracic and 28 lumbar. Conditions included osteo-arthritis, disc lesions, senile osteoporosis, ankylosing spondylitis, trauma and backache secondary to pathology at the hip.

The medical group included general medical (salpingitis, diabetis, psoriasis, bedsores, etc.)-15, neurlogical (polyneuritis, peripheral nerve lesions, trigeminal herpes, etc.)-24, Bell's palsy -7 , rheumatiod arthritis-9, osteo-arthritis-8. Of the patients with Bell's palsy, five recovered completely The treatment given was ultrasound (less than .5 watts $/ \mathrm{cm}^{2}$ for 3-5 minutes) during the stage of pain. As soon as voluntary movements returned, the treatment used was ice and exercises using stretch, resistance and irradiation from the normal side, neck and arms. One had pain for one month and was admitted for decompression. The other had had the attack 2 years previously and wanted to try the "new technique". He had partially recovered and showed no further recovery. This group did not include facial paralysis due to stab-wounds or cerebro-vascular accidents.

This is not the only general clinic where the students gain practical experience. They attend at other hospitals and specialised centres, and work in the gymnasium and wards at the Johannesburg Hospital.

B. WINTER, M.C.S.P. Teacher of Physiotherapy 